\section{Counterpoint: Appropriately Ambiguous-The Disc Osteophyte Complex}

\author{
(D).S. Ross, AJNR Editor-in-Chief
}

$\mathbf{T}$ he nomenclature for lumbar spine degenerative disc disease has been painstakingly conceived, revised, and condoned by numerous societies. ${ }^{1}$ Not so for the cervical spine. I would like to promote the term "disc osteophyte complex" (DOC) for standard usage in the cervical spine lexicon, with certain caveats.

\section{Why DOC?}

Cervical spondylosis is unique to the cervical spine because of the anatomy and presence of the uncovertebral joints and spinal cord. This is often manifest over several segments with mass effect on the ventral thecal sac and cord from ventral disease (DOC) and posterior ligamentous hypertrophy. On MR imaging, we are often faced with a low-signal, posterior extension of the disc margin, which compresses or effaces the dura and neural structures. This low-signal-intensity lesion may be composed of various aspects of disc degeneration, including a bulging annulus, disc herniation, osteophyte, cartilage, and calcification, which are not easily separated by routine MR imaging. Differences in image quality due to section thickness, magnet strength, and patient cooperation make more specific parsing of the images impossible. It is reasonable and prudent to use a term that is morphologically accurate, but also purposefully nonspecific for the large number of cases in which we just cannot tell the precise underlying (and microscopic) pathology.

\section{Surgeons}

Ideally, we would craft our reports about cervical spondylosis on the basis of an intimate knowledge of the practice patterns and training of the surgeons with whom we work. The reality is that we often dictate with limited clinical information and little knowledge of the physicians the individual patient might end up consulting, be it family practice, pain management, or neuro- and

http://dx.doi.org/10.3174/ajnr.A4928 orthopedic surgery. Opinions among surgeons vary widely regarding whether surgery should be performed for specific clinical indications, and if it should be performed, which approach (anterior or posterior) should be used (Figure). ${ }^{2}$ Given the vagaries involved, it seems reasonable to use nomenclature that is simple, easy, and widely understandable while also focusing on the important descriptors such as alignment, the degree of cord compression, and cord signal intensity. In the end, if the referring physicians are concerned about the presence of calcifications or osteophytes before surgery, they can always get a CT and correlate that data with the MR imaging study.

\section{Caveats}

1) Disc osteophyte complex should not be used in the lumbar spine. The pathophysiology is different, so stick to using the standard nomenclature of bulge, osteophyte, and disc herniation.

2) DOC should not be used to the exclusion of the terms "osteophyte" and "disc herniation" (protrusion, extrusion) when they are obvious in the cervical spine. Some patients will have a plump herniation showing high signal on gradient-echo, and these should be called "herniations." Some patients will have obvious osteophytes with fatty marrow signal, so go ahead and call them "osteophytes." Many, many patients, however, will end up having DOC!

3) DOC should be part of the analysis for central canal stenosis and separate from the analysis of uncovertebral joint and foraminal narrowing.

4) DOC is an abbreviation for this editorial only. If you use this term in your reports, please expand and use "disc osteophyte complex."

It is time to embrace the ambiguity of the DOC and use it guilt-free as standard nomenclature in cervical spondylosis.

\section{REFERENCES}

1. Fardon DF, Williams AL, Dohring EJ, et al. Lumbar disc nomenclature: version 2.0-recommendations of the combined task forces of the North American Spine Society, the American Society of Spine Radiology, and the American Society of Neuroradiology. Spine (Phila Pa 1976) 2014;39:E1448 - 65 CrossRef Medline

2. Lee JY, Hohl JB, Fedorka CJ, et al. Surgeons agree to disagree on surgical options for degenerative conditions of the cervical and lumbar spine. Spine (Phila Pa 1976) 2011;36:E203-12 CrossRef Medline
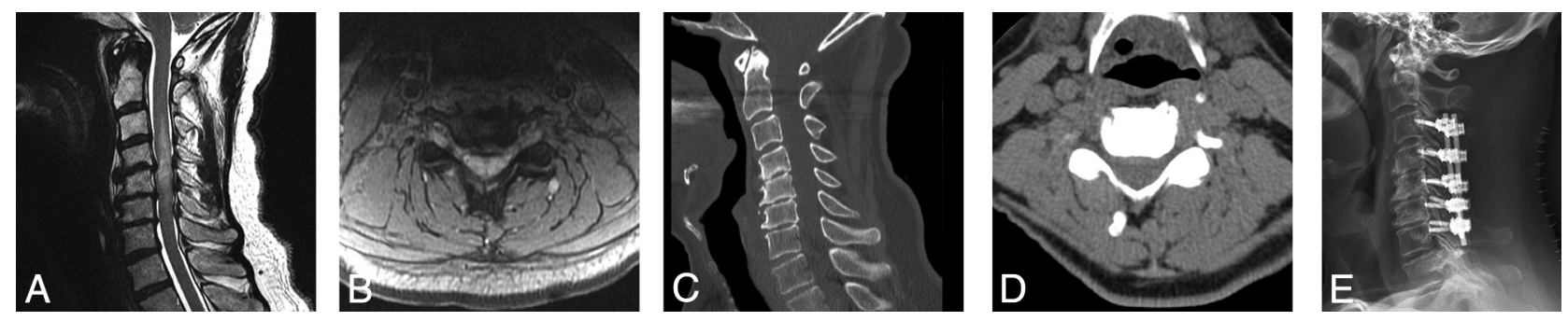

FIGURE. A 65-year-old patient with upper extremity numbness and paresthesias. An MR imaging study performed first ( $A$ and $B$ ) shows a low-signal DOC effacing the cord, with a long segment of cord myelomalacia. Nonenhanced CT study (C and D) obtained preoperatively shows that the bulk of the anterior disease is not ossified. Nevertheless, the patient went on to undergo posterior decompression (E). 